\title{
Hearing loss and diabetes mellitus
}

Inflammation, hyperglycemia and neuropathic or microvascular factors may contribute to diabetes-related hearing loss, according to a new study by US scientists. "The intent of the analysis was to investigate which pathological mechanisms might be responsible for this greater occurrence of hearing impairment in persons with diabetes," says investigator Kathleen Bainbridge.

Data from population-based studies has shown that diabetes mellitus is associated with hearing loss but the mechanisms that contribute to this impairment are unknown.

Bainbridge and colleagues investigated whether vascular or neuropathic conditions, cardiovascular risk factors, glycemia or inflammation mediate the association between diabetes mellitus and impaired hearing. The researchers analyzed the US NHANES data from 1999-2004 in 1,508 people aged $40-69$ years who had completed audiometric testing.

Bainbridge et al. observed that people with diabetes mellitus were twice as likely to have hearing impairment at low or mid frequences $(500,1,000$ and 2,000 Hz) than people who did not have the disease when adjusting for factors such as age, sex and occupational noise exposure. They also found evidence that inflammation, peripheral neuropathy, cochlear vasculopathy, hyperglycemia-but not hypertension or dyslipidemia-might mediate the relationship between diabetes mellitus and hearing impairment.

"We did find some preliminary evidence for neuropathic, microangiopathic, and inflammatory pathways [for diabetesrelated hearing impairment]," adds Bainbridge, who hopes to establish a temporal relationship between diabetes mellitus and hearing impairment using longitudinal studies.

\section{Katrina Ray}

Original article Bainbridge, K. E. et al. Potential mediators of diabetes-related hearing impairment in the U.S. population-NHANES 1999-2004. Diabetes Care doi:10.2337/dc09-1193 